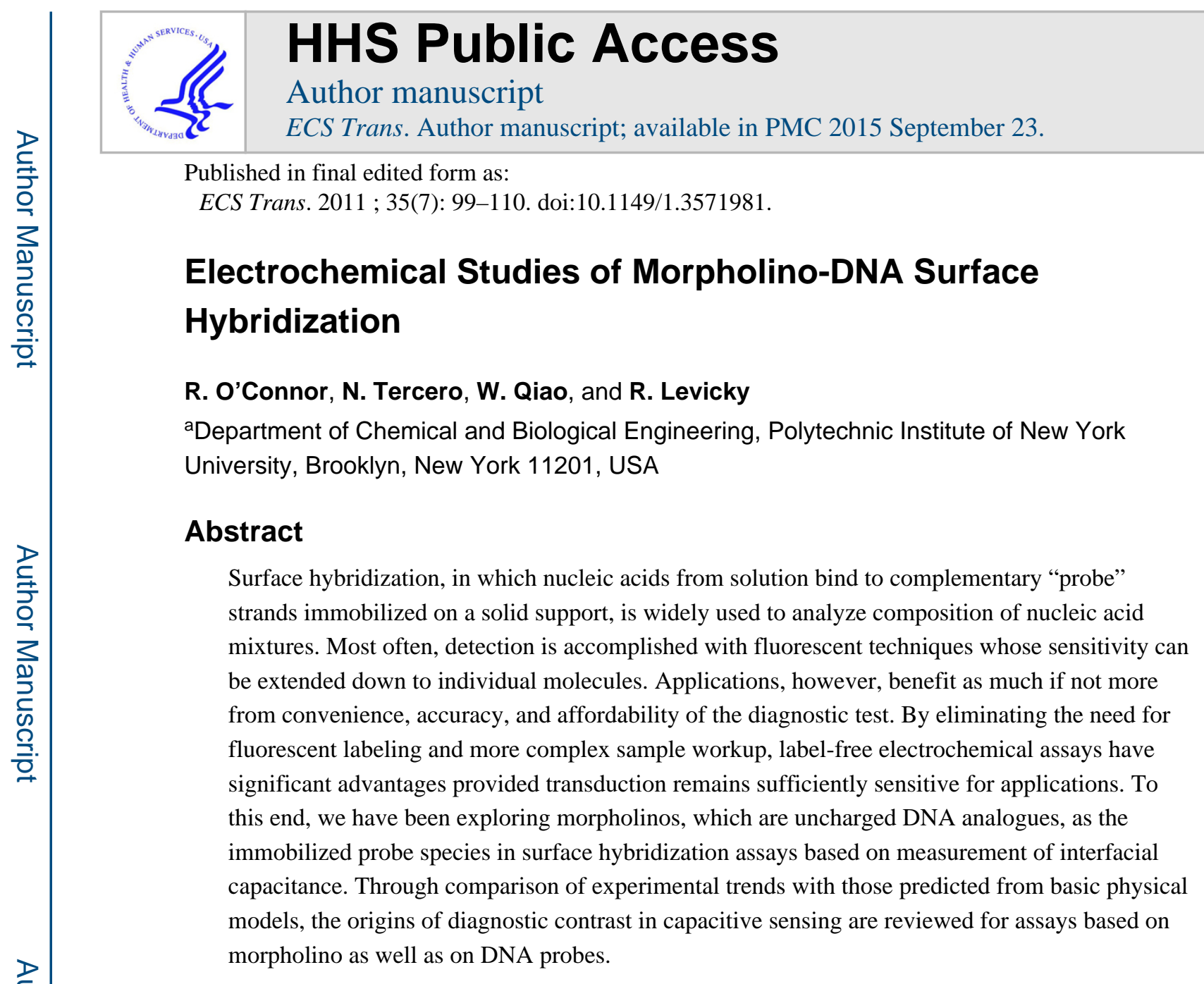

\title{
Introduction
}

Detection of biomolecular binding can be conveniently realized through electrostaticallymediated phenomena with techniques that include field effect (1-2) and impedance (3-6) methods. In these instances, molecular binding leads to changes in charge concentrations and/or polarizability in the vicinity of the sensing interface, and these changes are detected directly without the requirement for analyte labeling. This class of methods has been successfully applied to DNA detection. Their sensitivity, however, is often fundamentally limited by the salt concentrations needed to overcome electrostatic repulsion between DNA analyte and the layer of probe DNA strands on the solid support. Although higher ionic strength facilitates binding of analyte to the like-charged probe layer, it also screens electrostatic communication between the layer and the underlying sensor and thereby weakens electrostatic transduction; therefore, more analyte must bind to realize the same diagnostic response.

One way to circumvent the electrostatic barrier to hybridization is to use, instead of nucleic acid probes, uncharged analogues which similarly hybridize targets in a sequence-specific manner but lack the electrical charge. Peptide nucleic acids (PNAs) (7-14) and morpholinos (MOs) (15-20) are examples of synthetic DNA analogues that have been used as assay probes. We have focused on MO probes because of their synthetic flexibility with regard to base composition and sequence length. MOs can hybridize with nucleic acids even in 
deionized water;(17) therefore, assays can be performed under much lower ionic strengths than with DNA probes, leading to increased sensitivity of electrostatically-mediated detection.(18)

This report compares measured and model-predicted changes in interfacial capacitance brought on by hybridization of surfaces modified with MO or DNA probes. The aim of this comparison is to clarify origins of capacitive transduction as rooted in changes in the interfacial molecular organization, and to illustrate why DNA and MO probes lead to qualitatively different responses. These insights are necessary to guide optimization of diagnostic response, for example, by identifying conditions under which different contrast mechanisms add rather than negate each other, so that the net hybridization signal is enhanced. Other efforts have aimed to connect diagnostic response in field-effect devices with a structural description of the sensing interface.(21-22)

\section{Experimental Summary}

Experimental data were taken from a prior study (17) in which 18mer single-stranded DNA targets of the sequence $5^{\prime}$ ATC ACT TGC AGA ATT TAA $3^{\prime}$ were hybridized to endimmobilized 20mer MO or DNA probes with the base composition 5' TTT TAA ATT CTG CAA GTG AT 3'. A $0.2 \mathrm{~mol} \mathrm{~L}^{-1} \mathrm{pH} 7.0$ phosphate buffer served as electrolyte and hybridization medium, with no other salts added. Target concentration was $25 \mathrm{nmol} \mathrm{L}-1$. Molarity is expressed in phosphate groups. Probe films were prepared in two steps: (1) chemisorption of the MO or DNA probe through a terminal disulfide moiety to the gold working electrode (1.6 mm diameter) followed by (2) passivation of the electrode with mercaptopropanol (MCP) in order to block nonspecific contacts between probe backbones and the gold. Figure 1 illustrates this general approach for the case of MO probes. By labeling the probes with electroactive ferrocene tags, cyclic voltammetry could be used to determine the total probe coverage $S_{0}$. $\mathrm{An} \mathrm{Ag} / \mathrm{AgCl} / 3 \mathrm{~mol} \mathrm{~L}^{-1} \mathrm{NaCl}$ electrode served as reference and a Pt wire was used as the counterelectrode. All experimental data are reported relative to the $\mathrm{AgCl}$ reference. The differential capacitance $C_{\mathrm{d}}$, used to monitor hybridization, was determined every 5 minutes using a frequency $f=5435 \mathrm{~Hz}$ and a $5 \mathrm{mV}$ ac rms amplitude. $C_{\mathrm{d}}$ follows from the out-of-phase impedance $Z^{\prime \prime}, C_{\mathrm{d}}=1 /\left(2 \pi f A\left|Z^{\prime \prime}\right|\right)$, where $A$ is the area of the electrode after correction for surface roughness.(23-24) $C_{\mathrm{d}}$ was determined as a function of applied dc bias $V_{\mathrm{DC}}$, with $V_{\mathrm{DC}}$ ranging from -0.2 to $0.25 \mathrm{~V}$. This experimental approach uses $V_{\mathrm{DC}}$ to establish the local ionic concentrations, and the superimposed ac sinusoid to read out the capacitive response at those conditions.

In addition, the 25mer probe sequence 5' GTA GCT AAT GAT GTG GCA TCG GTT G 3', derived from the macrophage infectivity potentiator (Mip) gene of Legionella pneumophila Philadelphia 1 (GenBank accession number AE017354), was used to demonstrate detection of 95 base pair targets 5' GCA ATG TCA ACA GCA ATG GCT GCA ACC GAT GCC ACA TCA TTA GCT ACA GAC AAG GAT AAG TTG TCT TAT AGC ATT GGT GCC GAT TTG GGG AAG AA 3' (sense strand). The double-stranded targets were generated by PCR amplification of the Mip gene with the forward primer 5' GCA ATG TCA ACA GCA ATG GCT $3^{\prime}$ and the reverse primer $5^{\prime}$ TTC TTC CCC AAA TCG GCA CC $3^{\prime}$. Two successive PCR reactions were performed to increase the concentration of amplicons. Both 
PCR reactions used Taq PCR Master Mix kits (Qiagen, Valencia, CA) and followed standard protocol from the vendor in a $100 \mu \mathrm{L}$ volume. Amplification started at $94{ }^{\circ} \mathrm{C}$ for 3 min, followed by 30 cycles each at $94{ }^{\circ} \mathrm{C}$ for $30 \mathrm{~s}, 52^{\circ} \mathrm{C}$ for $45 \mathrm{~s}$, and $72^{\circ} \mathrm{C}$ for $30 \mathrm{~s}$. The first PCR used templates of genomic DNA isolated from Legionella pneumophila Philadelphia I strain grown axenically in liquid culture using standard DNA preparation protocols, while the second used $1 \mu \mathrm{L}$ of products from the first amplification. PCR amplicons were checked on $1.5 \%$ agarose gel by electrophoresis and purified using MinElute PCR Purification kits (Qiagen). The concentration of thus purified DNA was measured on a Cary 50 spectrophotometer. Deposition of MO probes on Au working electrodes was for $40 \mathrm{~min}$ at a concentration of $8 \mu \mathrm{mol} \mathrm{L}^{-1}$, followed by $150 \mathrm{~min}$ immersion in $1 \mathrm{mmol} \mathrm{L}^{-1}$ solution of MCP. After rinsing in deionized water, working electrodes were immersed in target-free hybridization buffer $\left(0.1 \mathrm{~mol} \mathrm{~L}^{-1} \mathrm{pH} 7.0\right.$ phosphate buffer $)$ prior to hybridization. Hybridization was performed using $0.5 \mu \mathrm{g} \mathrm{ml}^{-1}\left(\sim 10 \mathrm{nmol} \mathrm{L}^{-1}\right)$ of the double-stranded target. During hybridization, $C_{\mathrm{d}}$ was determined every 5 minutes using a frequency $f=2604 \mathrm{~Hz}$ and a $5 \mathrm{mV}$ ac rms amplitude with $V_{\mathrm{DC}}$ ranging from -0.2 to $0.05 \mathrm{~V}$.

\section{Numerical Modeling}

Figure 2A illustrates the structural model used for MO probe films. Because of limited solubility, unhybridized MO probes (Fig. 2A green regions) exist in a desolvated, precipitated state on the solid support. $(17,25)$ Ions can enter the desolvated layer under the influence of an electric field applied through the working electrode. Because probe coverage $S_{0}$ was in a range $\left(S_{0}<\sim 5 \times 10^{12} \mathrm{~cm}^{-2}\right)$ where consumption of probes by hybridization is expected to render the layer discontinuous,(18) patches of unhybridized probes coexist laterally with solvent-filled voids. More soluble MO-DNA duplexes (Fig. 2A blue regions) protrude past the unhybridized layer into the solvent. As discussed below, the earlier model (18) was slightly modified in that duplexes are not assumed to be perpendicular to the solid support; rather, they are approximated as possessing the average tilt predicted for unhindered rotation about the tethering points. The MCP layer (Fig. 2A brown region) lies between the working electrode and the probe layer and, due to its dense molecular packing, is interpreted as impermeable to solution ions.

Figures 2B and 2C show two different structural models considered for DNA films. Since even unhybridized DNA probes are polyelectrolytes with good solubility, they were assumed to coexist side-by-side with DNA-DNA duplexes. A separate layer of collapsed, unhybridized probes is therefore not present. Hybridized DNA-DNA duplexes were approximated as tilted, same as for MO-DNA duplexes. Additionally, experiments indicated that pure $\mathrm{MCP}$ films (see below) exhibit a $V_{\mathrm{DC}}$-dependent capacitive response consistent with permeability to ions at the layer's outer periphery, in the vicinity of the terminal $-\mathrm{OH}$ groups; therefore, in Figure 2C a thin, ion-permeable portion has been added on top of an impermeable MCP core. Interestingly, for MO probe films such a permeable portion was not needed to capture experimental trends; this simplification is attributed to permeability of the desolvated layer of unhybridized MO probes, which has a similar impact on calculations of $C_{\mathrm{d}}$. 
The above structural models were used in conjunction with a numerical calculation of $C_{\mathrm{d}}$ based on a one-dimensional Poisson-Boltzmann (PB) framework described elsewhere. (1718) In this approach, each separate layer in the model (e.g. impermeable MCP; permeable $\mathrm{MCP}$, if present; desolvated MO probes, if present; duplexes) is assigned a dielectric constant $\varepsilon$, a thickness $t$, a concentration of immobilized charge $c_{\mathrm{im}}$, and a partitioning penalty $\beta_{\mathrm{j}}$ for ions $j$ present in phosphate buffer, namely $\mathrm{Na}^{+}, \mathrm{H}_{2} \mathrm{PO}_{4}{ }^{-}, \mathrm{HPO}_{4}{ }^{2-}$, and $\mathrm{PO}_{4}{ }^{3-}$. The $\beta_{\mathrm{j}}$ represent energetic costs in excess of electrostatic work, measured in units of $k T$, to transport a $j$ type ion from bulk solution into a layer. The partitioning penalties may reflect, for example, changes in ionic solvation. Ionic concentrations in bulk solution were calculated for $\mathrm{pH} 7.0$ conditions using phosphate $\mathrm{p} K_{\mathrm{a}}$ values of $2.15,6.87$, and 12.3. The PB calculations incorporate several approximations. Foremost, these include (1) treatment of ions as point charges, (2) neglect of changes in layer structure (e.g. duplex orientation) in response to $V_{\mathrm{DC}}$, (3) assumption that properties of each layer are uniform perpendicular as well as parallel to the solid support, and (4) neglect of the effect of internal ionic concentrations on a layer's dielectric constant and organization.

Table I summarizes the model inputs used for hybridization of $20 \mathrm{mer}$ probes with $18 \mathrm{mer}$ targets. For samples based on MO probes, the MCP layer was modeled as before (17-18) with $\varepsilon=4.4$, thickness $t$ as determined by MCP molecular dimensions and packing density, and impermeability to all ions enforced by high, $\beta=1000$, values. For the layer of unhybridized MO probes, $\varepsilon$ was estimated from the Bruggeman effective medium expression (26-27)

$$
\phi_{1} \frac{\varepsilon_{1}-\varepsilon}{\varepsilon_{1}+2 \varepsilon}+\left(1-\phi_{1}\right) \frac{\varepsilon_{2}-\varepsilon}{\varepsilon_{2}+2 \varepsilon}=0
$$

where $\varphi_{1}$ is the volume fraction of MO probes plus MO-DNA duplexes, both of which are taken to possess a pure state static permittivity of $\varepsilon_{1}=9.1,(17-18)$ and $\left(1-\varphi_{1}\right)$ is the volume fraction of the solvent voids with $\varepsilon_{2}=80$ corresponding to water near $20^{\circ} \mathrm{C}$. For the MO layer, the volume fraction includes contributions from unhybridized probes $\varphi_{\mathrm{P}}$ as well as portions of MO-DNA duplexes $\varphi_{\mathrm{D}}$,

$$
\phi_{1}=\phi_{P}+\phi_{D}=\frac{S_{P}}{S_{P}^{*}}+\frac{a_{D} l_{D}}{\left(\left(l_{D} \cos \theta\right) / S_{D}\right)}
$$

where $S_{\mathrm{P}}=S_{0}-S_{\mathrm{D}}$ is coverage of remaining unhybridized probes and $S_{\mathrm{D}}$ is the coverage of MO-DNA duplexes. $S_{\mathrm{P}}{ }^{*}=5 \times 10^{12} \mathrm{~cm}^{-2}$ is the minimum probe coverage required for a continuous film, corresponding to a pure MO layer 0.53 nm thick.(18) For $S_{\mathrm{P}}$ below $S_{\mathrm{P}}^{*}$, as in the present experiments, the unhybridized MO layer is therefore modeled as maintaining a thickness of $0.53 \mathrm{~nm}$ as hybridization proceeds but with an increasing fraction of solvent voids; in this situation the unhybridized probes contribute a volume fraction $\varphi_{\mathrm{P}}$ equal to $S_{\mathrm{P}} /$ $S_{\mathrm{P}} *$. The second term on right in equation $2 \mathrm{a}$ corresponds to the duplex volume fraction $\varphi_{\mathrm{D}}$, given by the ratio of duplex volume $a_{\mathrm{D}} l_{\mathrm{D}}$, where $a_{\mathrm{D}}=3.14 \times 10^{-14} \mathrm{~cm}^{2}$ is the duplex crosssectional area and $l_{\mathrm{D}}=6.1 \times 10^{-7} \mathrm{~cm}$ is its length, to the available interfacial volume per duplex. The available interfacial volume, in the denominator, consists of the product of the 
thickness of the duplex layer $l_{\mathrm{D}} \cos \theta$ where $\theta=57^{\circ}$ is the average duplex tilt angle from the surface normal, and the interfacial area per duplex equal to the reciprocal of $S_{\mathrm{D}}$. The values used for $a_{\mathrm{D}}$ and $l_{\mathrm{D}}$ assumed MO-DNA duplexes to be structurally similar to B-form doublestranded DNA, and the average tilt angle is based on a uniform orientational probability distribution of a rod immobilized by one end to an impenetrable flat support. Although interactions of duplexes with each other and with charges on the support are expected to bias their orientation, these effects are neglected.

The $\beta$ values for the unhybridized MO layer are the same as used previously. (17-18) In this regard, comparison of predicted with experimental trends suggested that only $\mathrm{Na}^{+}$and $\mathrm{H}_{2} \mathrm{PO}_{4}{ }^{-}$be allowed to partition into the desolvated MO layer, as partitioning of $\mathrm{HPO}_{4}{ }^{2}$ caused model-predicted $C_{\mathrm{d}}$ to exhibit an overly strong dependence on $V_{\mathrm{DC}}$ when compared with experiment. Physically, the exclusion of multiply ionized phosphate species could reflect difficulty in maintaining multiple negative charges per phosphate in the low dielectric environment of the MO layer. Lastly, the immobilized charge concentration $c_{\mathrm{im}}$ arises from MO-DNA duplexes, which are taken to partially localize within the probe layer as drawn in Figure 2A. $c_{\text {im }}$ is estimated from

$$
c_{i m}=\frac{\left|z_{D}\right|}{\left(l_{D} \cos \theta\right) / S_{D}} \quad \text { [3] }
$$

where $z_{\mathrm{D}}=-11$ is the valence of MO-DNA duplexes after correcting for counterion condensation,(18) and the denominator is the interfacial volume per duplex as in equation 2a.

Equation 1 is also used to estimate $\varepsilon$ of the MO-DNA duplex layer, but now only duplexes contribute to $\varphi_{1}$ so that

$$
\phi_{1}=\frac{a_{D} l_{D}}{\left(\left(l_{D} \cos \theta\right) / S_{D}\right)}
$$

For purposes of calculation the thickness of the duplex layer is restricted to three Debye lengths $r_{\mathrm{D}}$, corresponding to $1.4 \mathrm{~nm}$ (Table I). This truncation was implemented because the rapid decay of electric fields within the layer precluded numerical integration across its full width. The impact of this truncation on calculated $C_{\mathrm{d}}$ values is less than a tenth of a percent. (18) Since the duplex layer is well-solvated and mostly comprised of solvent, no penalties were applied to ion partitioning so that all $\beta_{\mathrm{j}}=0$. Equation 3 is again used for $c_{\mathrm{im}}$.

Modeling of samples based on DNA probes required a more complex representation of the MCP layer. Figure 3 compares experimental $C_{\mathrm{d}}$ data from a pure MCP layer as a function of $V_{\mathrm{DC}}$. Interpretation of the MCP layer as strictly impermeable to ions resulted in qualitative disagreement between prediction and experiment, with a much flatter predicted response (Figure 3, dashed line). In comparison, allowing penetration of $\mathrm{Na}^{+}$and $\mathrm{H}_{2} \mathrm{PO}_{4}{ }^{-}$in the vicinity of the MCP hydroxyl headgroups, by lowering the corresponding $\beta$ values from 1000 to 1 , led to much improved agreement (Figure 3 solid line). Accordingly, a two layer 
MCP description was used for DNA probe samples, consisting of an impermeable MCP core and a permeable peripheral region as in Figure $2 \mathrm{C}$, and with the inputs listed in Table I. That a two-layer MCP description was not necessary in the case of MO probes is attributed to presence of the desolvated probe layer, whose ionic permeability rendered the further addition of such a feature to the MCP layer unnecessary.

Due to their aqueous solubility, unhybridized DNA probes were taken to remain solvated and therefore to coexist with hybridized duplexes as a single layer. The $\varepsilon$ of this layer was estimated from equation 1 , with the DNA volume fraction $\varphi_{1}$ given by

$$
\phi_{1}=\frac{v_{R E S}\left(S_{D} N_{D}+S_{P} N_{P}\right)}{l_{D} \cos \theta} \quad[2 \mathrm{c}]
$$

where $v_{\text {RES }}=0.53 \mathrm{~nm}^{3}$ is the average volume of a nucleotide residue, and $N_{\mathrm{D}}=38$ and $N_{\mathrm{P}}=$ 20 are the numbers of residues in duplexes and probes, respectively. The duplex length $l_{\mathrm{D}}$ and average tilt angle $\theta$ were taken to have the same values as for MO-DNA duplexes. For simplicity $\varepsilon_{1}=9.1$ was assumed to apply both to DNA probes and to DNA-DNA duplexes. This value is intended to account also for water of hydration surrounding the DNA strands. Because of its good solvation the DNA layer was assumed permeable to all ions, and the immobilized charge concentration was estimated using

$$
c_{i m}=\frac{\left(S_{D}\left|z_{D}\right|+S_{P}\left|z_{P}\right|\right)}{l_{D} \cos \theta}
$$

where $z_{\mathrm{D}}=-12.9$ and $z_{\mathrm{P}}=-7.4$ were taken from a recent hybridization study of this same probe-target system.(28)

\section{Capacitive Response to Hybridization}

The structural models of the previous section, in conjunction with the PB solution methodology described earlier,(17-18) were used to predict the capacitive response to hybridization for $\mathrm{MO}$ and DNA probe layers as a function of $V_{\mathrm{DC}}$ and duplex coverage $S_{\mathrm{D}}$. These predictions were compared to experimental data to better understand how organizational changes in the layer, induced by hybridization, translate to a capacitive signal useful for diagnostic applications. The experimental response consists of the change in capacitance, $\Delta C_{\mathrm{d}}$, attributed to formation of duplexes on the working electrode. Experimental values for $\Delta C_{\mathrm{d}}$ were obtained by first correcting raw $C_{\mathrm{d}}$ data for baseline drift, followed by subtraction of $C_{\mathrm{d}}$ prior to hybridization from values determined after hybridization commenced. The baseline drift is attributed to gradual desorption of the MCP molecules.(18)

Figure $4 \mathrm{~A}$ plots raw $C_{\mathrm{d}}$ vs $V_{\mathrm{DC}}$ traces at various times for hybridization of $18 \mathrm{mer}$ DNA targets to 20mer MO probes, with red curves measured pre-hybridization and black traces measured while hybridization progressed, following introduction of $25 \mathrm{nmol} \mathrm{L}^{-1}$ target. The upward displacement of the $C_{\mathrm{d}}\left(V_{\mathrm{DC}}\right)$ traces with time during pre-hybridization is due to 
baseline drift, and can be mathematically represented by the function $f_{B}=A_{1}-A_{2} \exp (-k t)$. (18) Fitting of $A_{1}, A_{2}$, and $k$ to the pre-hybridization data at each $V_{\mathrm{DC}}$, followed by subtraction of the time-dependent portion, removed effects of the drift and collapsed the data as in Figure 4B. Only the pre-hybridization data superimpose, as hybridization introduces both a sideways shift to higher $V_{\mathrm{DC}}$ in the capacitive minimum as well as an additional upward displacement of the $C_{\mathrm{d}}\left(V_{\mathrm{DC}}\right)$ curves. $\Delta C_{\mathrm{d}}$ is obtained by subtracting the last measured red trace from the hybridized curves. The same processing algorithm was applied to calculation of $\Delta C_{\mathrm{d}}$ when DNA probes were used. With DNA probes, the displacement in capacitive minimum to higher $V_{\mathrm{DC}}$ remained although it was smaller in magnitude; however, in contrast to MO probes, hybridization induced a downward rather than an upward shift in the curves.(17)

Figure 5A shows experimental $\Delta C_{\mathrm{d}}$ calculated from the MO probe data of Figure 4, while Figure 5B shows data for hybridization of 95 base pair double-stranded PCR amplicons to $25 \mathrm{mer}$ MO probes (see Experimental Summary). In both cases, hybridization leads to a positive $\Delta C_{\mathrm{d}}$ that diminishes in magnitude at more positive $V_{\mathrm{DC}}$. Although the $V_{\mathrm{DC}}$ range sampled in Figure 5B is narrower, it is clear that a qualitatively similar response is observed for the much longer double-stranded target as for the single stranded oligonucleotide. These results indicate that the contrast mechanisms responsible for $\Delta C_{\mathrm{d}}$ are not specific to a unique sequence or target. An inversion in contrast is observed in Figure 5A at the most positive $V_{\text {DC }}$. In contrast, DNA-DNA surface hybridization, Figure 5C, does not exhibit a positive $\Delta C_{\mathrm{d}}$ at any of the tested biases; instead only a weaker, increasingly negative $\Delta C_{\mathrm{d}}$ response is observed as $V_{\mathrm{DC}}$ increases. The probe and target sequences in Figures $5 \mathrm{~A}$ and $5 \mathrm{C}$ were identical, and the probe coverages were the same within experimental uncertainty.

Figure 6 presents predictions for $\Delta C_{\mathrm{d}}$ calculated from the models described under Numerical Modeling, for the case of $18 \mathrm{mer}$ targets hybridizing to $20 \mathrm{mer}$ MO (Figure 6A) or DNA (Figure 6B) probes. These calculations used inputs corresponding to the data in Figures 5A and $5 \mathrm{C}$, respectively. For the calculations, $S_{\mathrm{D}}$ was varied to cover the full range of possible conversion $x=S_{\mathrm{D}} / S_{0}$ from 0 to 1 , as plotted on the $y$-axes in Figure 6 . In comparison, for the experimental data in Figure 5 hybridization progressed to some $x$ less than full conversion, with the precise value not known since $S_{\mathrm{D}}$ was not determined. Accordingly, the experimental $\Delta C_{\mathrm{d}}$ are plotted against time rather than $x$. From further experiments) with labeled targets, $(17,29)$ experimental conversion for the MO-DNA hybridization is expected to reach $\sim 0.3$ while that for the DNA-DNA system is expected to approach $\sim 0.5$. Another difference between Figures 5 and 6 is that $V_{\mathrm{DC}}$ in the experiments is expressed relative to the reference electrode, while for the calculations it is expressed relative to bulk solution. For these reasons the discussion will focus on qualitative comparison of trends.

An examination of Figures 5 and 6 reveals that experimental trends are reasonably reproduced by the calculations. For MO-DNA surface hybridization, both experimental and predicted $\Delta \underline{C}_{\mathrm{d}}$ responses are positive at more negative $V_{\mathrm{DC}}$ but eventually decrease as $V_{\mathrm{DC}}$ rises, leading to contrast inversion at the most positive biases considered. The calculations also indicate that if hybridization progresses to sufficiently high conversions the contrast inversion disappears, so that positive $\Delta C_{\mathrm{d}}$ values are recovered. The experiments do not show such recovery - this is attributed to hybridization not reaching the high conversions 
required. For the DNA-DNA system, both experiment and calculations indicate that $\Delta C_{\mathrm{d}}$ is negative, with the decrease in capacitance more prominent at more positive $V_{\mathrm{DC}}$. Overall, MO probes provide a significantly enhanced response to hybridization than DNA probes. Since the models evidently contain sufficient detail to qualitatively reproduce experimental trends, it should be possible to rationalize experimental observations in terms of physical phenomena incorporated in the models.

The two principal sources of capacitive changes in the models are variations in permittivity and in ionic concentrations at the surface. For MO probes, the dominant permittivity effect stems from a decrease in coverage of unhybridized, desolvated probes - as these probes hybridize and become more soluble, solvent is able to more closely approach the solid support causing an increase in the near surface permittivity and hence contributing to a positive $\Delta C_{\mathrm{d}}$. This " $\varepsilon$-effect" applies to all $V_{\mathrm{DC}}$ settings. The impact of hybridization on $\Delta C_{\mathrm{d}}$ through the ionic concentrations is more complex. Hybridization causes an increase in the negative, immobilized target charge at the surface. The requirement of near electroneutrality of the surface region can then be satisfied either through accumulation of cations, or the expulsion of anions. Which of these mechanisms dominates depends on $V_{\mathrm{DC}}$. At negative biases, the interface is enriched in cations while the local concentration of anions is low. Therefore, anion expulsion can only be a secondary effect, and hybridization predominantly results in further accumulation of cations and an increase in the local ionic strength. Since higher ionic strength corresponds to more effective screening of electric fields, it contributes to a positive $\Delta C_{\mathrm{d}}$. In contrast, at sufficiently positive biases the interface is rich in anions and deficient in cations. Under these conditions accumulation of target charge is principally compensated through anion expulsion; since the expulsion lowers the local ionic strength it contributes negatively to $\Delta C_{\mathrm{d}}$.

Based on the above considerations negative $V_{\mathrm{DC}}$ settings are generally more favorable when MO probes are used. This is because, for negative $V_{\mathrm{DC}}$, changes in ionic concentrations and in permittivity both contribute positively to $\Delta C_{\mathrm{d}}$; therefore, the two contrast mechanisms add to enhance the overall signal. On the other hand, at sufficiently positive $V_{\mathrm{DC}}$ the two mechanisms are opposed, with permittivity contributing positively to $\Delta C_{\mathrm{d}}$ but ionic effects contributing negatively. This partial cancellation weakens the overall response. Further calculations show that the recovery of positive $\Delta C_{\mathrm{d}}$ in Figure 6A at high $x$ can be primarily attributed to increase in the dielectric constant $\varepsilon$ of the probe layer as $x$ nears full conversion, with a secondary impact due to switch from cation-poor to cation-rich conditions driven by accumulation of negative target charge.

In contrast to MO probes, DNA probes are well solvated even in the unhybridized state. In this case, the impact of hybridization on permittivity is primarily to displace solvent from the interface to make room for target molecules. This exchange results in a permittivity decrease, since permittivity of DNA is less than that of the aqueous solvent, and thus a negative contribution to $\Delta C_{\mathrm{d}}$. The qualitative impact of changes in ionic concentrations remains unchanged from that observed with MO probes, although it is significantly weakened in magnitude. Therefore, when DNA probes are used optimal conditions for capacitive detection arise at positive $V_{\mathrm{DC}}$, since then both permittivity and ionic effects contribute in the same sense to $\Delta C_{\mathrm{d}}$ to provide maximum (negative) response to 
hybridization. On the other hand, at negative $V_{\mathrm{DC}}$ the two contrast mechanisms nearly cancel, so that little response is evidenced even as hybridization proceeds (Figures $5 \mathrm{C}$ and $6 \mathrm{~B})$.

\section{Acknowledgments}

This project was supported by Award No. R33HG003089 from the National Human Genome Research Institute, National Institutes of Health, USA.

\section{References}

1. Yang W, Hamers RJ. Appl Phys Lett. 2004; 85:3626.

2. Fritz J, Cooper EB, Gaudet S, Sorger PK, Manalis SR. Proc Natl Acad Sci U S A. 2002; 99:14142. [PubMed: 12386345]

3. Daniels JS, Pourmand N. Electroanalysis. 2007; 19:1239. [PubMed: 18176631]

4. Berggren C, Stålhandske P, Brundell J, Johansson G. Electroanalysis. 1999; 11:156.

5. Vagin MY, Trashin SA, Karyakin AA, Mascini M. Anal Chem. 2008; 80:1336. [PubMed: 18205333]

6. Mearns FJ, Wong ELS, Short K, Hibbert DB, Gooding JJ. Electroanalysis. 2006; 18:1971.

7. Macanovic A, Marquette C, Polychronakos C, Lawrence MF. Nucleic Acids Res. 2004; 32:e20. [PubMed: 14739233]

8. Uno T, Tabata H, Kawai T. Anal Chem. 2007; 79:52. [PubMed: 17194121]

9. Liu JY, Tian SJ, Nielsen PE, Knoll W. Chem Commun. 2005; 2969

10. Degefa TH, Kwak J. J Electroanal Chem. 2008; 612:37.

11. Aoki H, Buhlmann P, Umezawa Y. Electroanalysis. 2000; 12:1272.

12. Keighley SD, Estrela P, Li P, Migliorato P. Biosens Bioelectron. 2008; 24:906. [PubMed: 18774288]

13. Wang J, Palecek E, Nielsen PE, Rivas G, Cai X, Shiraishi H, Dontha N, Luo D, Farias PAM. J Am Chem Soc. 1996; 118:7667.

14. Fang Z, Kelley SO. Anal Chem. 2009; 81:612. [PubMed: 19086897]

15. Wang X, Smirnov S. ACS Nano. 2009; 3:1004. [PubMed: 19284734]

16. Gao Z, Ting BP. Analyst. 2009; 134:952. [PubMed: 19381390]

17. Tercero N, Wang K, Gong P, Levicky R. J Am Chem Soc. 2009; 131:4953. [PubMed: 19296583]

18. Tercero N, Wang K, Levicky R. Langmuir. 2010; 26:14351. [PubMed: 20690772]

19. Zhang GJ, Luo ZH, Huang MJ, Tay GK, Lim EJ. Biosens Bioelectron. 2010; 25:2447. [PubMed: 20435462]

20. Li SJ, Li J, Wang K, Wang C, Xu JJ, Chen HY, Xia XH, Huo Q. ACS Nano. 2010; 4:6417. [PubMed: 20958077]

21. Landheer D, Aers G, McKinnon WR, Deen MJ, Ranuarez JC. J Appl Phys. 2005; 98:art. no. 044701.

22. McKinnon WR, Landheer D, Aers G. J Appl Phys. 2009; 104:art. no. 124701.

23. Shen G, Tercero N, Gaspar MA, Varughese B, Shepard K, Levicky R. J Am Chem Soc. 2006; 128:8427. [PubMed: 16802807]

24. Oesch U, Janata J. Electrochim Acta. 1983; 28:1237.

25. Gong P, Wang K, Liu Y, Shepard K, Levicky R. J Am Chem Soc. 2010; 132:9663. [PubMed: 20572663]

26. Bruggeman DAG. Annal Phys (Leipzig). 1935; 416:636.

27. Landauer R. J Appl Phys. 1952; 23:779.

28. Irving D, Gong P, Levicky R. J Phys Chem B. 2010; 114:7631. [PubMed: 20469913]

29. Gong P, Levicky R. Proc Natl Acad Sci U S A. 2008; 105:5301. [PubMed: 18381819] 


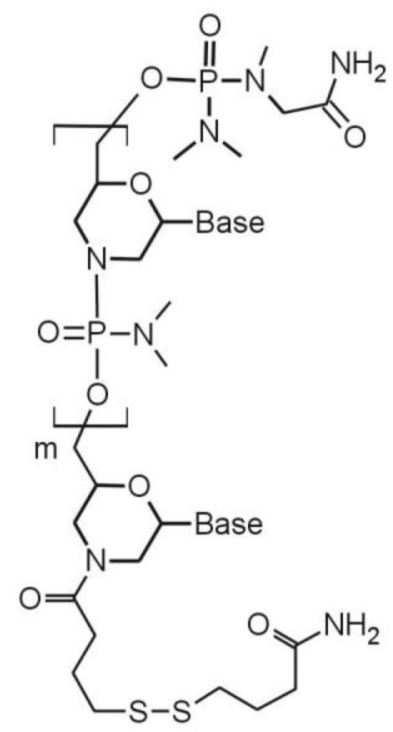

A
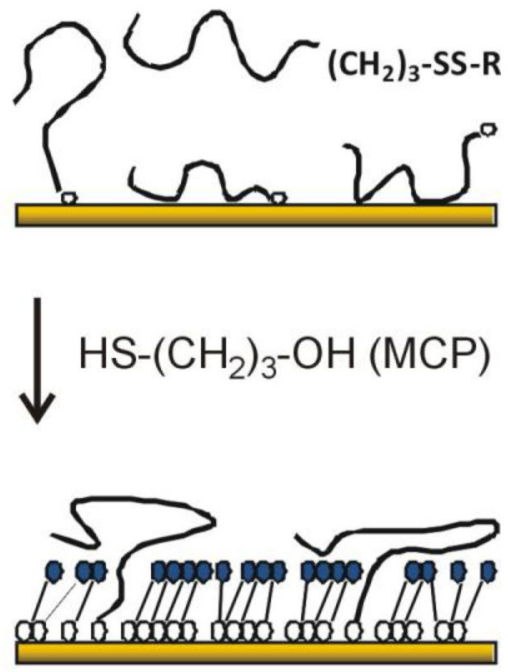

B

Figure 1.

(A) Structure of a morpholino probe. The disulfide end was used for immobilization to gold working electrodes. (B) Schematic of probe layer preparation. Probes were chemisorbed first, followed by surface passivation with mercaptopropanol (MCP). 


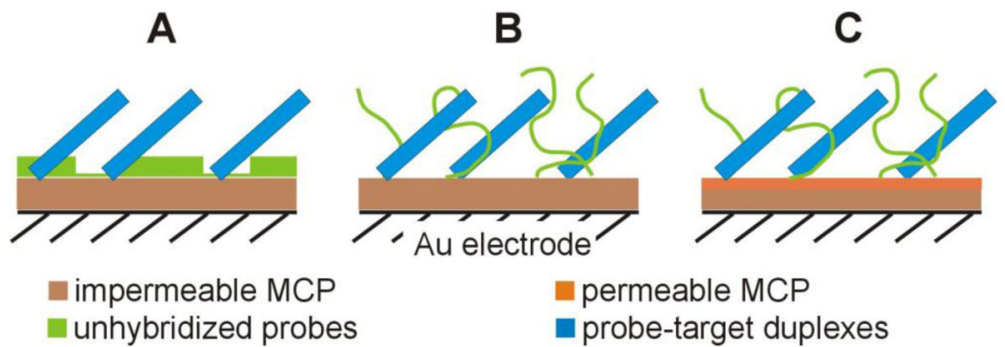

Figure 2.

Structural models used to describe (A) MO and (B)-(C) DNA probe films. See text for discussion. 


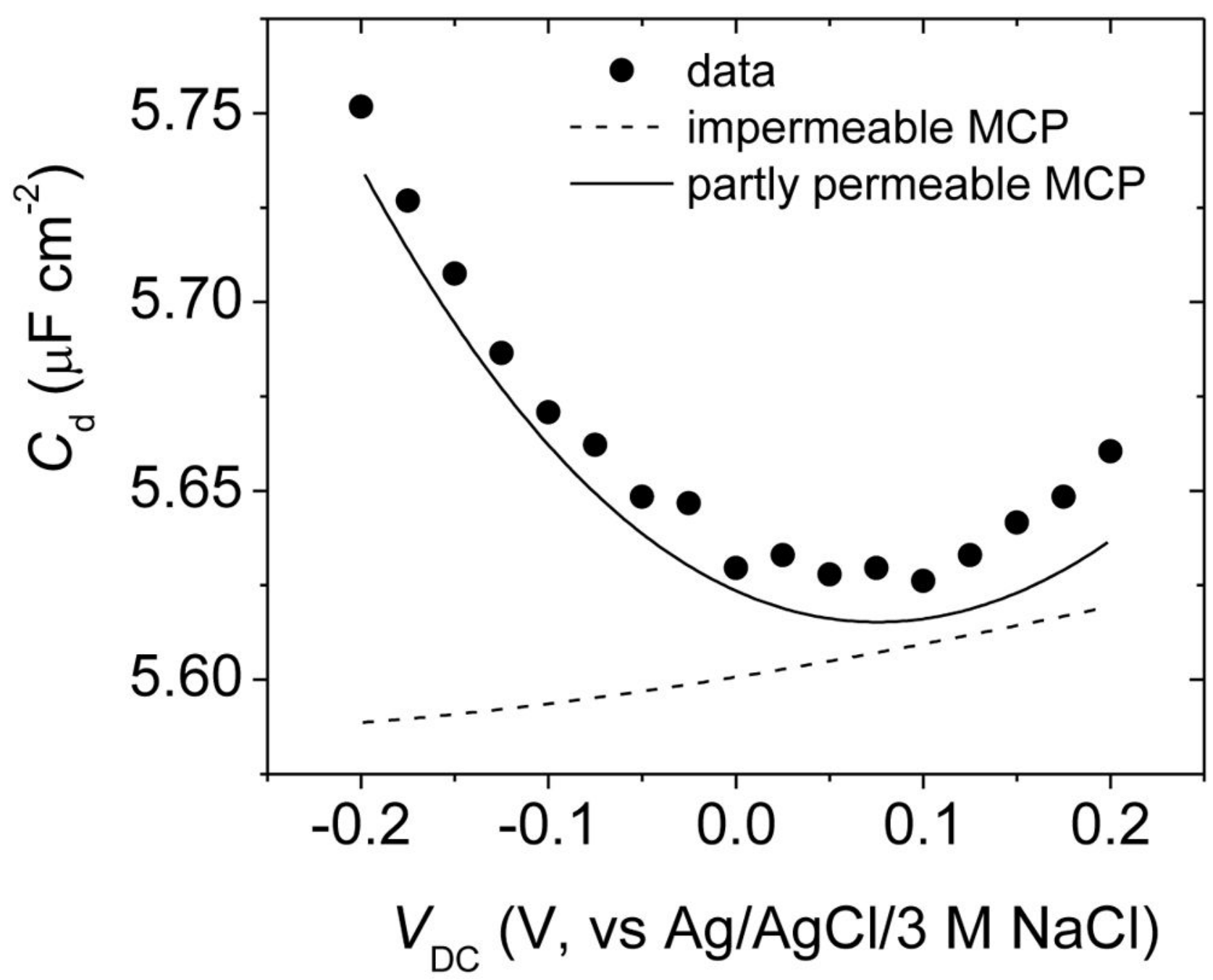

Figure 3.

Comparison of experimental $(\bigcirc) C_{\mathrm{d}}\left(V_{\mathrm{DC}}\right)$ data from a pure MCP layer to model predictions based on an impermeable description of the MCP layer (-) and a partly permeable description in which ions are allowed to penetrate a thin portion near the MCP headgroups (- -), see Table I. Electrolyte: $0.2 \mathrm{~mol} \mathrm{~L}^{-1} \mathrm{pH} 7.0$ phosphate buffer. 

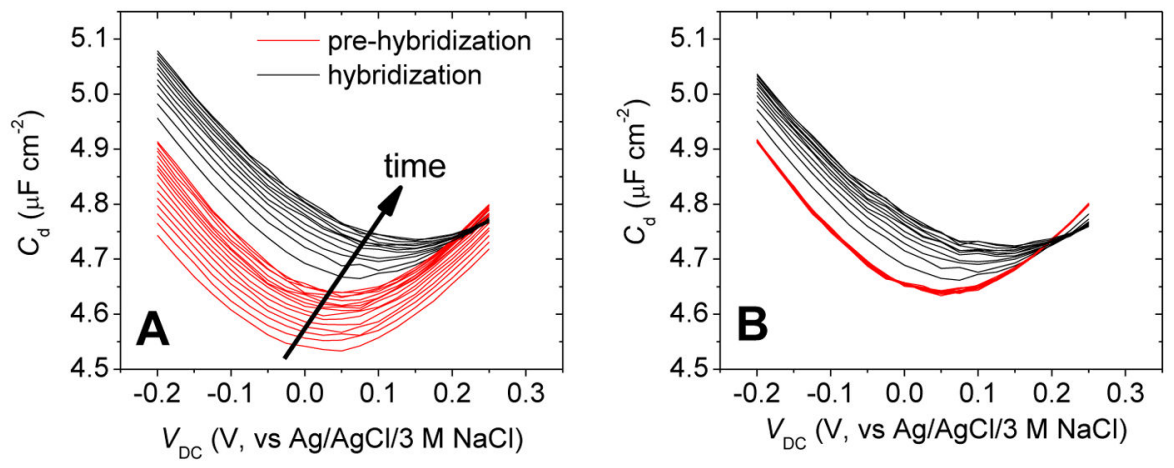

Figure 4.

(A) Raw $C_{\mathrm{d}}\left(V_{\mathrm{DC}}\right)$ data from hybridization of 18 mer DNA targets to 20 mer MO probes, in $0.2 \mathrm{~mol} \mathrm{~L}^{-1} \mathrm{pH} 7.0$ phosphate buffer and for $S_{0}=4.9 \times 10^{12} \mathrm{~cm}^{-2}$. Base sequences are listed in the Experimental Summary. (B) Same data after correction for baseline drift. 


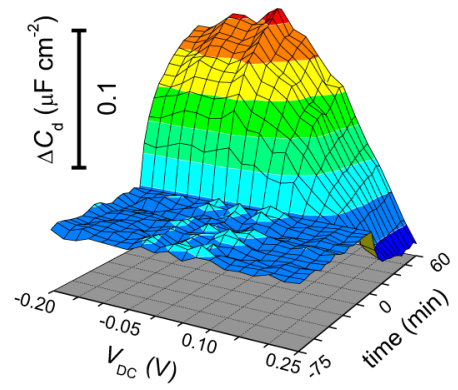

A

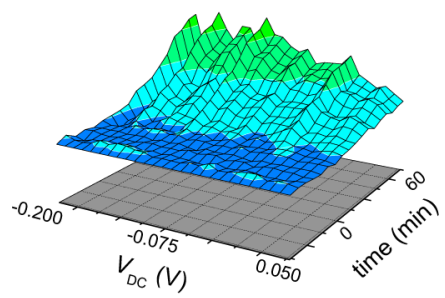

B

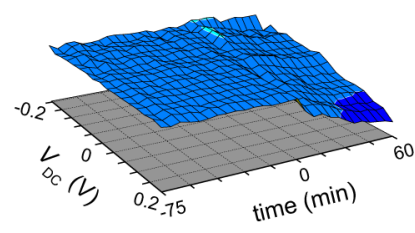

C

Figure 5.

Experimental capacitive response for hybridization between (A) 18mer single-stranded DNA targets and 20mer MO probes $\left(S_{0}=4.9 \times 10^{12} \mathrm{~cm}^{-2}\right)$, (B) $95 \mathrm{mer}$ double-stranded DNA targets and 25mer MO probes $\left(S_{0}=3.1 \times 10^{12} \mathrm{~cm}^{-2}\right)$, and (C) $18 \mathrm{mer}$ single-stranded DNA targets and 20mer DNA probes $\left(S_{0}=5.1 \times 10^{12} \mathrm{~cm}^{-2}\right)$. Strand sequences and hybridization conditions at described in the Experimental Summary. The same vertical $\Delta C_{\mathrm{d}}$ scale, shown in (A), applies to all three figures. 


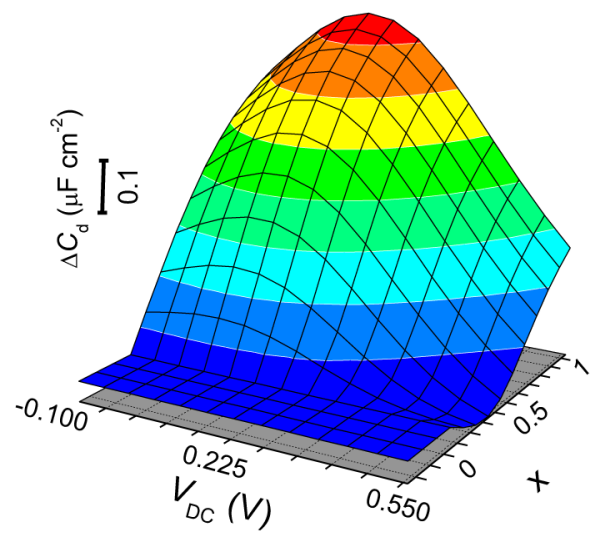

A

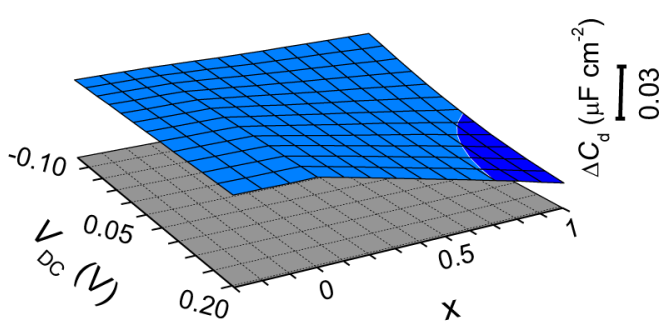

B

Figure 6.

Model predictions for the capacitive response to hybridization between (A) 18mer singlestranded DNA targets and 20mer MO probes $\left(S_{0}=4.9 \times 10^{12} \mathrm{~cm}^{-2}\right)$ and (B) $18 \mathrm{mer}$ singlestranded DNA targets and 20mer DNA probes $\left(S_{0}=5.1 \times 10^{12} \mathrm{~cm}^{-2}\right)$. On the $y$-axes, $x$ represents hybridization conversion given by $x=S_{\mathrm{D}} / S_{0}$. 

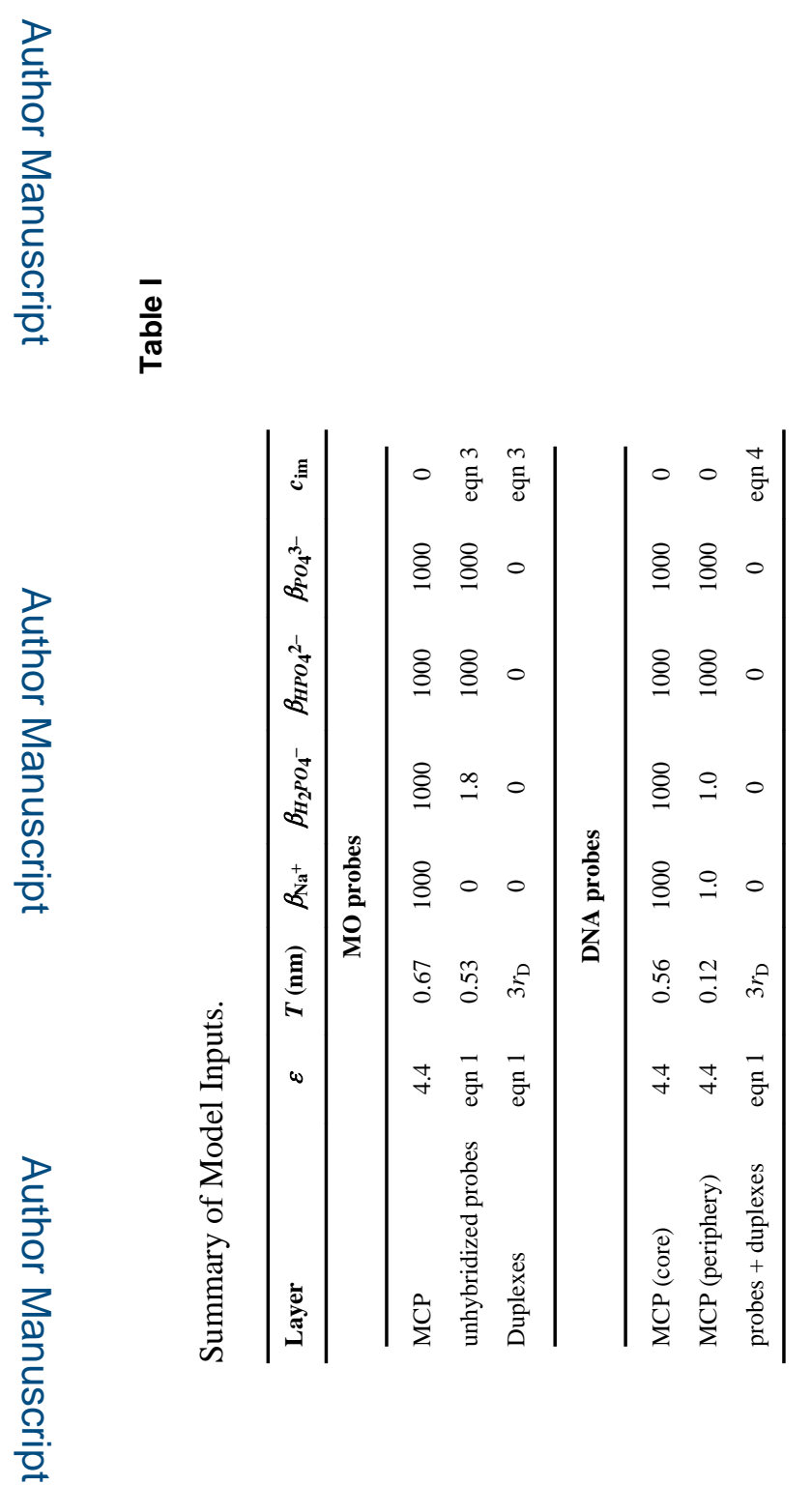